\title{
L'information scientifique, un enjeu de société
}

L'année 1998 a vu l'affrontement, dans un combat dont l'arbitre était la population suisse toute entière, d'une communauté scientifique et de forces décidées à interdire le développement d'un pan essentiel de la recherche biologique. Aucun pays n'est à l'abri de tels projets politiques visant à empêcher certaines recherches. La communauté scientifique suisse a su se faire comprendre de façon magistrale, comme $\mathrm{m} / \mathrm{s}$ l'a rapporté avec enthousiasme au mois de juin dernier. Une leçon s'impose : c'est en informant le plus largement possible la population sur leurs recherches et leurs méthodes de travail, que les scientifiques suisses ont réussi à répondre à l'attaque. Les Suisses ont ainsi, en quelques mois, reçu de leurs scientifiques une véritable information, dépourvue de sensationalisme, sur ce qu'est la recherche biomédicale, ses objectifs, ses difficultés, ses réussites mais aussi ses échecs. Ces échanges avec une population entière et sa formation aux réalités de la recherche scientifique, sont sans doute les résultats les plus heureux de cet épisode.

Les formes de la démocratie suisse, qui autorisent la tenue d'un tel référendum d'initiative populaire sur la base d'une demande appuyée par 100000 signatures, n'existent pas dans la plupart des autres pays du monde. Cela ne veut pas dire que le problème de l'information scientifique de la population ne se pose pas dans les mêmes termes. Bien au contraire, le référendum suisse doit servir de révélateur et renforcer l'idée que les scientifiques, s'ils veulent continuer à obtenir son soutien, doivent toujours avoir à cœur de transmettre leur savoir à la population, de la faire bénéficier le plus largement possible des connaissances nouvelles qu'ils ont pour tâche d'acquérir. Cette transmission n'est toutefois pas réalisable directement, du moins dans la plupart des cas. Elle s'effectue le long d'une chaîne dont tous les maillons sont d'égale importance : les enseignants, à tous les niveaux, les journalistes de la presse et des médias généralistes, dont l'impact est aujourd'hui de plus en plus important dans le domaine des sciences, comme enfin ceux qui nous concernent plus particulièrement, les médecins confrontés quotidiennement aux questions des patients et de leurs familles. Pour que les acquis de la connaissance fondent une amélioration du niveau culturel général de la société, les scientifiques doivent savoir s'adresser à ces différents professionnels pour que ceux-ci, dans les domaines et dans les termes qui leur sont propres, informent le grand public.

médecine/sciences tente d'occuper, sur ce terrain de la transmission scientifique, la place du premier maillon. En parallèle de notre rôle de média multidisciplinaire de l'actualité de la recherche biomédicale à destination de la communauté scientifique, notre objectif est en effet de fournir aux universitaires et aux médecins les éléments de la recherche biologique en train de s'accomplir, pour leur permettre d'envisager aussi complètement que possible les enrichissements futurs de leur enseignement et de leur pratique. Notre singularité, qui définit la position que nous occupons, est que l'ensemble des textes qui paraissent dans médecine/ sciences sont rédigés par des chercheurs, biologistes et cliniciens, acteurs directs de la recherche dont ils sont, mieux que tous autres, à même de rapporter les résultats, mais aussi les difficultés, les interrogations, les perspectives. Pour accomplir cette tâche, notre revue se félicite d'avoir compté cette année 316 auteurs dans sa partie magazine, outre les dizaines de scientifiques français et québécois mais aussi belges et suisses qui composent les comités de médecine/ sciences, et d'avoir recueilli l'avis de 213 arbitres différents, pour 121 synthèses et mini-synthèses publiées (voir les listes, accompagnées de nos remerciements cordiaux, page 1469 du $n^{\circ} 12$, décembre 1998 et page 4 de ce numéro.

Cette participation massive de la communauté scientifique francophone à notre revue est la démonstration que médecine/sciences répond à une ou, plutôt, à des attentes. Celle, d'abord, d'une lecture de la recherche biologique et médicale de la plus grande qualité, car tel est notre souci premier et constant. Celle d'une présentation rigoureuse de ses résultats et perspectives, ouverte mais dépourvue du sen- sationnalisme ou de l'emphase déplacés que l'on trouve malheureusement trop dans certains médias. Celle, enfin, de l'utilisation de notre langue pour l'élaboration d'un discours scientifique riche de toutes ses nuances en même temps qu'accessible.

L'année de médecine/sciences a été marquée par plusieurs changements, que nos lecteurs n'auront pas manqué de remarquer. L'arrivée de nombreux membres nouveaux dans les deux comités éditoriaux, et la responsabilité entière donnée à Élisabeth Bursaux de la partie magazine de la revue ont permis de l'enrichir et de la diversifier. La désignation de Rémi Quirion, à partir de janvier 1999, comme référent québecois pour le magazine sera encore un autre atout. La rubrique "Histoire de la Médecine et des Sciences", à présent étendue sous le titre d'Histoire et Sciences Sociales, a pris un nouvel essor sous la conduite de trois membres des comités éditoriaux, spécialisés dans ces domaines. Nous croyions pouvoir donner cette année un accès ouvert à $\mathrm{m} / \mathrm{s}$ sur Internet. Cela s'est révélé problématique à l'éditeur, même pour l'index dont ce serait, pourtant, la place. Nous ne pouvons qu'espérer pour l'année qui vient cette extension sur la «toile», qui ouvrirait bien plus efficacement, notamment, notre revue aux scientifiques de langue latine, en Europe et en Amérique latine, auxquels le français scientifique et médical est parfaitement accessible. Enfin, et nous terminerons sur cette note optimiste, la contribution de la communauté scientifique belge à notre revue s'est renforcée d'année en année et nous avons été particulièrement heureux de publier, en mai dernier, un troisième numéro de $\mathrm{m} / \mathrm{s}$ consacré à l'une des grandes Universités belges francophones $(\mathrm{m} / \mathrm{s}$ vol. 14, $n^{\circ} 5$ ). C'est dans cette voie d'une extension toujours plus grande de médecine/sciences au service des communautés scientifiques francophones que nous continuerons à œuvrer.

Que l'année 1999 soit, pour tous, riche d'enthousiasmes, de découvertes et de réussites !

Marc Peschanski Rédacteur en chef, Paris Michel Bergeron Rédacteur en chef, Montréal 